\title{
Anomalous behavior of dispersion curves in water-like systems and water
}

\author{
Yu. D. Fomin, E. N. Tsiok, V. N. Ryzhov, and V. V. Brazhkin ${ }^{1}$ \\ 1 Institute for High Pressure Physics RAS, 108840 Kaluzhskoe shosse, 14, Troitsk, Moscow, Russia
}

(Dated: December 27, 2018)

\begin{abstract}
In the present paper we consider dispersion curves of longitudinal excitations of a model coresoftened liquid and SPC/E model of water. We show that both systems demonstrate anomalous behavior of the excitation frequencies: the frequencies of the excitations can decrease with temperature along isochors, while in normal liquids they should increase. This observation allows to introduce one more water anomaly - anomalous dependence of excitation frequencies on temperature.

PACS numbers: $61.20 . \mathrm{Gy}, 61.20 . \mathrm{Ne}, 64.60 . \mathrm{Kw}$
\end{abstract}

\section{INTRODUCTION}

It is widely known that many properties of crystals can be efficiently described in frames of their collective excitations - phonons [1, 2]. The phonon approach allows to predict many important properties of crystalline solids such as, for instance, the stability of a crystal or its heat capacity. In this respect it is also important to mention that investigation of collective excitations makes a link between dynamic and thermodynamic properties of matter.

The situation becomes more complex in the case of liquids. Although the collective excitations in liquids were extensively studied both theoretically [3] and experimentally [4], the absence of a simple exactly solvable model like the model of a harmonic crystal, prevented the appearance of a theory of liquid based on the collective excitations. Only few years ago some publications appeared which tried to explain the behavior of the heat capacity of liquids basing on collective excitations [5]. Although the results presented in this paper are very encouraging, this topic definitely requires further development.

Two topics on collective excitations in liquids got most of attention. First of all, it is the existence of transverse excitations in liquids. It was believed for a long time that liquids do not support the transverse excitations. At the same time it was widely known that liquids with very high viscosity do demonstrate transverse waves. Moreover, later it was recognized that even the liquids with low viscosity can sustain transverse excitations. For example, in Ref. [6] dynamical properties of Lennard-Jones model were discussed. The results of this paper predicted the existence of transverse excitations in the system. Nowadays the transverse excitations were observed experimentally in liquids metals [7-12] and some other liquids. So the existence of transverse waves can be considered as strictly proved.

Another point of interest is the so called positive sound dispersion (PSD). The PSD means that the frequency of longitudinal excitations exceeds the frequency obtained from the Debye law $\omega_{D}(k)=c_{s} \cdot k$, where $c_{s}$ is the adiabatic speed of sound. PSD also was observed experi- mentally in many different systems, for instance, liquid metals [7-12]. Importantly, both PSD and transverse excitations disappear when the temperature is risen or the density is decreased. It allowed to propose that these properties can be used to demarcate liquid-like and gaslike regimes in fluids $13-15$.

Even further complication can be expected in the case of so called "anomalous liquids", i.e. the liquids which demonstrate unusual properties. The most well known anomalous liquid is water which demonstrates more then 70 anomalies, for instance, density anomaly, diffusion anomaly, maxima of thermodynamic response functions, etc (one can find an extensive description of most of the anomalous properties of water at the site [16]). One can see that water demonstrates both thermodynamic and dynamic anomalies. However, up to now we are not aware of any systematic research of possible anomalous behavior of collective excitations in water.

Although the behavior of water is of extreme interest it appears to be very complex. Up to now there is no any model of water which can correctly describe many different properties (for example, phase diagram, viscosity, the location of density maxima, etc.) simultaneously. In Refs. [17, 18] a critical comparison of several common water models is given. The authors give the highest scores to the TIP4P/2005 model [27], however, even this model strongly underestimates the melting temperatures at different pressures [17].

For the reason of the difficulties described above it is often reasonable to start investigations from simple models which can demonstrate some properties similar to water, for example, density or diffusion anomaly. Investigation of such systems can give a deep insight into the nature of some phenomena observed in water and other anomalous liquids being computationally cheaper then studies of the water itself. In Ref. 19] physical origin of polyamorphism of ice is discussed. A simple model able to reproduce the coexistence of two disordered phases was proposed. In this model two tetrahedra of water (4 molecules complexes) interact with each other via a coresoftened potential. Although core-softened systems fail to reproduce the behavior of water exactly they are able 
to mimic many unusual water properties, such as density anomaly, diffusion anomaly, structural anomaly, maxima of the response functions, etc.

Core-softened systems received a lot attention in the literature. Many different systems were proposed and studied. It was found that these systems demonstrate numerous unusual features: complex phase diagram, maxima on the melting line, unusual phases (for example, diamond phase in a system with isotropic pair potential [20, 24]), water-like anomalies, etc. Moreover, the behavior of the system is very sensitive to the shape of the interaction potential which allows to modify the system in order to reproduce a feature of interest. For example, in Ref. 22] it was shown that the regions of density, diffusivity and structural anomalies in water form nested domains in the density-temperature plane. In Ref. 23] the same work was performed for liquid silica and it was discovered that the mutual location of the anomalies is different from the case of water. In Refs. [24, 25] a system with a core-softened potential was studied. The functional form of the potential was remained constant, but its parameters varied. It was shown that in frames of the same model one can obtain the location of the anomalous regions like in water or like in silica. Similar effects of changing of some anomalous regions are observed when comparing different tetrahedral liquids, for example, water, silicon and germanium modeled by Stillinger-Weber potentials 26.

A model which demonstrates complex behavior with numerous anomalies was introduced in our previous works [23, 24]. Below we will call it Repulsive Shoulder System (RSS) model. This is a system of particles interacting via a potential is given by the formula:

$$
U(r) / \varepsilon=\left(\frac{\sigma}{r}\right)^{14}+0.5\left(1-\tanh \left(k\left(r-\sigma_{1}\right)\right)\right),
$$

with $k=10.0$ and the parameter $\sigma_{1}$ determines the width of the repulsive shoulder. It is convenient to express all quantities in the units of the potential, i.e. the parameter $\varepsilon$ is used as a unit of energy and $\sigma$ as a unit of length. All other quantities can be expressed from these parameter. Below all quantities are given in these reduced units. In our previous publications it was shown that this system demonstrates very complex behavior [23 28]. Moreover, the behavior of the system is extremely sensitive to the magnitude of the parameter $\sigma_{1}$. In particular, if $\sigma_{1}=$ 1.35 then the system demonstrates the diffusion anomaly, the density anomaly and the structural anomaly. In the case of $\sigma_{1}=1.55$ the diffusion anomaly disappears and with $\sigma_{1}=1.8$ the density anomaly also disappears 27 .

In the present paper we investigate the dispersion curves of longitudinal excitations of the RSS system and show that they demonstrate anomalous behavior: while in normal liquid the excitation frequency increases upon isochoric heating, in RSS the excitation frequency can decrease with isochoric temperature increase. We demonstrate that this anomalous behavior cannot be reproduced in the simplest theoretical approximation Hubburd-Beeby model which is widely used for description of the collective excitations in liquid metals. Finally, we perform preliminary study of dispersion curves of water and show that they also demonstrate the same anomaly.

\section{SYSTEM AND METHODS}

We simulated a system of 4000 particles in a cubic box with periodic boundary conditions interacting via the potential (1). We performed simulations along three distinct isochores: $\rho=0.5, \rho=0.65$ and $\rho=1.15$. The first isochore crosses the region of the water-like anomalies of the system. The second isochore is at slightly higher density then the anomalies reported earlier. Finally, the $\rho=1.15$ isochore belongs to the high-density regime where no anomalies are expected. The temperatures varies from $T_{\min }=0.1$ up to $T_{\max }=1.0$ at $\rho=0.5$, from $T_{\min }=0.3$ up to $T_{\max }=1.5$ at $\rho=0.65$ and from $T_{\min }=2.0$ up to $T_{\max }=10.0$ at $\rho=1.15$.

The system was simulated by means of molecular dynamics method. Firstly the system was equilibrated in NVT (constant number of particles N, volume V and temperature T) ensemble for $10 \cdot 10^{6}$ steps. The time step was set to $d t=1 \cdot 10^{-4}$. After the equilibration the system was propagated in microcanonical NVE (constant number of particles $\mathrm{N}$, volume $\mathrm{V}$ and internal energy $\mathrm{E}$ ) ensemble. In case of $\rho=0.5$ the propagation period is $2 \cdot 10^{8}$ steps. At other densities it is $1 \cdot 10^{8}$ steps. In order to improve the statistics we divide the propagation periods into the blocks of $5 \cdot 10^{6}$ steps and perform block averaging [33].

In order to find out the dispersion curves we calculated the longitudinal part of the velocity flux correlation function:

$$
C_{L}(k, t)=\frac{k^{2}}{N}\left\langle J_{z}(\mathbf{k}, t) \cdot J_{z}(-\mathbf{k}, 0)\right\rangle
$$

where $J(\mathbf{k}, t)=\sum_{j=1}^{N} \mathbf{v}_{j} e^{-i \mathbf{k r}_{j}(t)}$ is the velocity current and the wave vector $\mathbf{k}$ is directed along the $\mathrm{z}$ axis 34, 35]. Note that the wave vector $\mathbf{k}$ is not related to the parameter $k$ of the interaction potential. We believe that this coincidence in notation does not lead to ambiguity. The excitation frequencies $\omega$ were determined as peaks of the Fourier transform of $C_{L}$.

In spite of large simulation time and rather good averaging the raw spectra are still noisy. In order to remove this noise we approximate the spectra by the following equation:

$$
\omega(k)=c_{s} k+b_{1} k^{5 / 2}+b_{2} k^{11 / 4}+b_{3} k^{23 / 8},
$$




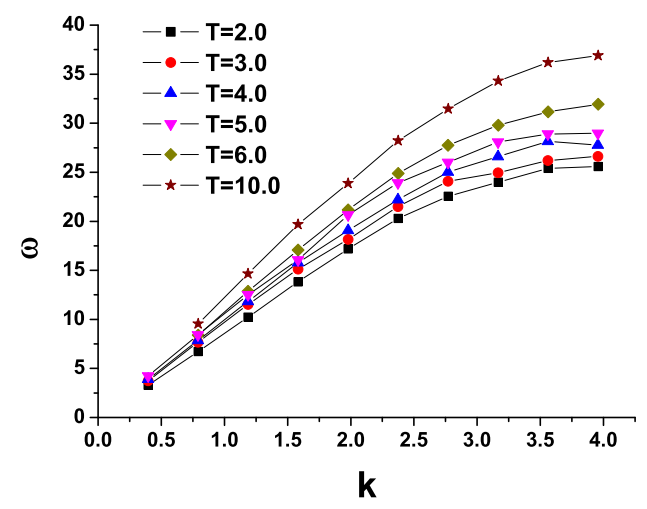

FIG. 1: Dispersion curves of the soft sphere system with $n=$ 12 at $\rho=1.0$ isochore.

where $c_{s}$ is adiabatic speed of sound and $b_{1}, b_{2}$ and $b_{3}$ are fitting coefficients [1]. This formula is obtained in frames of Mode-Coupling Theory (MCT) treatment of collective excitations in fluids. The adiabatic speed of sound was calculated as $c_{s}=\left(\gamma\left(\frac{\partial P}{\partial \rho}\right)_{T}\right)^{1 / 2}, \gamma=c_{P} / c_{V}$, $c_{P}=c_{V}+\frac{T}{\rho^{2}}\left(\frac{\partial P}{\partial T}\right)_{\rho}^{2}\left(\frac{\partial P}{\partial \rho}\right)_{T}^{-1}, c_{P}$ and $c_{V}$ are isobaric and isochoric heat capacities.

We also perform simulation of SPC/E model of water. We study the system of 4000 water molecules in a cubic box under PBC. The system is equilibrated for $0.5 \mathrm{~ns}$ in NVT ensemble. The time step is set to $0.1 \mathrm{fs} .2$ blocks of $3 \cdot 10^{6}$ steps are made for calculating of averages. NVE ensemble is used at this stage The calculation of the dispersion curves is made in the same fashion as in the case of RSS system.

We also give a plot of the dispersion of soft sphere system, i.e. the system of particles interacting via power law potential $U_{\text {soft }}=\varepsilon\left(\frac{\sigma}{r}\right)^{n}$. This system is a generic model of a simple liquid and it does not demonstrate any kind of anomalies. The simulation setup of this system is given in our previous publication [14].

Lammps simulation package is used for all simulations of this paper [38].

\section{RESULTS AND DISCUSSION}

In the beginning of the discussion we give example of the dispersion curves of soft sphere system with $n=12$ at $\rho=1.0$ and a set of temperatures (Fig. 1). Soft spheres is a system which can serve as etalon os a simple liquids, and no anomalies is expected in soft spheres. One can see that in a simple liquid the frequencies of excitations increase with temperature. Below we call this type of dependence as normal while decrease of the frequencies with temperature as anomaly.
Let us consider the dispersion curves of RSS at the density $\rho=0.5$ and different temperatures. As it is shown in the supplementary materials the dispersion curves are well fitted by the eq. 4. Therefore, here we show only the fitted curves.

Fig. 2 (a) shows the dispersion curves of the system at $\rho=0.5$ and a set of temperatures. One can clearly see that, for instance, the curve at $T=0.4$ is below the one at $T=0.3$. It means that the frequency decreases with temperature. However, if the temperature is increased further the frequencies start to increase with temperature as in a normal liquid. This behavior is qualitatively different from the one of soft spheres shown above. Fig. 2 (b) demonstrates the temperature dependence of the excitation frequency at fixed magnitude of the wave vector. Although the data are still a bit noisy one can clearly identify a minimum at the temperature around $T=0.5$. Therefore, one can say that the excitation spectra of the system are anomalous if from $T=0.3$ (the lowest temperature we studied) up to $T=0.5$ and behave like in normal liquid above this temperature.

We believe that anomalous dependence of the dispersion curves on temperature requires also theoretical interpretation. The simplest approach to the excitation spectra of liquids is the Hubbard-Beeby (HB) model [2]. However, it does not reproduce the anomalous temperature dependence of the frequencies (see the supplementary materials for the discussion). More elaborate theories are required.

We remind that at $\rho=0.5$ the system demonstrates the density anomaly [23 28] which leads to appearance of a minimum on the pressure dependence on temperature at this isochore. However, the adiabatic speed of sound $c_{s}$ along the same isochore is a monotonous function of temperature (see the supplementary materials for the plots of the $P$ and $c_{s}$ along the isochore). In this respect it is interesting to consider the PSD in this system. PSD is the phenomenon when the frequency $\omega(k)$ exceeds the Debye value $\omega_{D}(k)=c_{s} k$. Therefore, in the present case we observe two tendencies: the frequencies become smaller while the speed of sound increases. However, at $T>0.5$ the frequencies change to increasing with temperature. Therefore, one can expect some kind of non-monotonous behavior of PSD.

In order to study the PSD in the system we consider the difference $\omega(k)-c_{s} k$ (Fig. 3). The presence of PSD means that this quantity has positive magnitude. One can see that the PSD disappears somewhere between $T=0.8$ and 0.9 , but reappears again at $T=1.0$. Therefore, the non-monotonous behavior of PSD does appear in this system. Such disappearance of PSD was already observed in our recent publication [37], however, no explanation was given in that work. Now we see that the reason of such complex behavior is the anomalous behavior of the excitation frequencies.

The same calculations were repeated for two more den- 

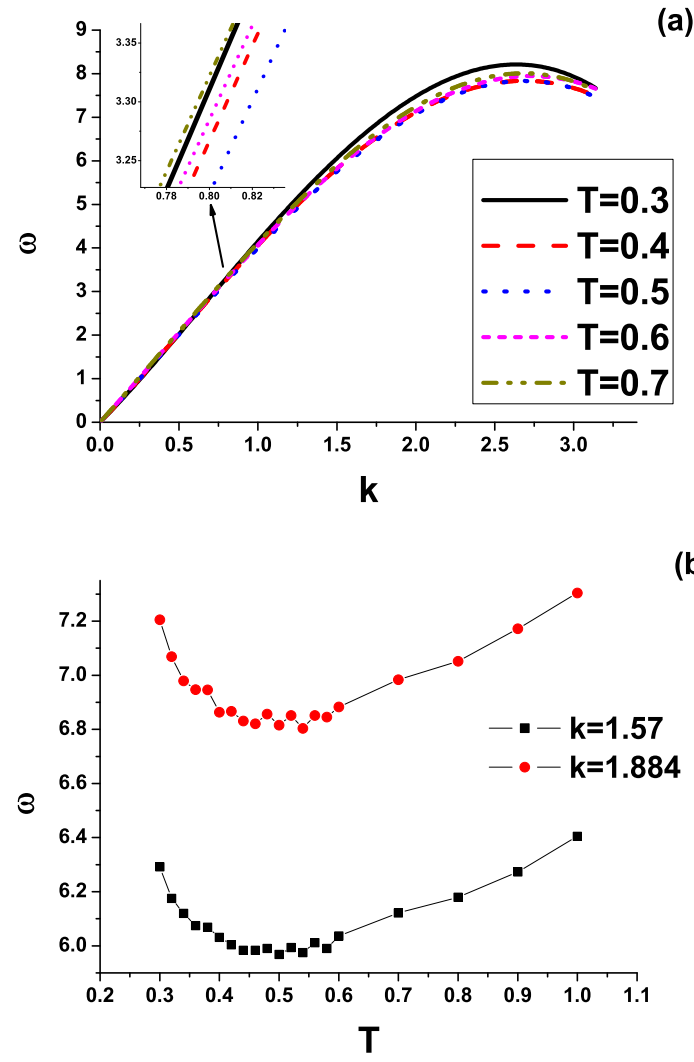

(b)

FIG. 2: (a) Dispersion curves of the system at $\rho=0.5$ which demonstrate the anomalous decrease of the frequency with temperature. The inset enlarges the region of $k$ around 0.8 . (b) Temperature dependence of the frequency at two magnitudes of the wave-vector $k$.

sities: $\rho=0.65$ and $\rho=1.15$. Fig. 4 4shows the dispersion curves (panel (a)), the temperature dependence of excitation frequencies at a fixed wave vector (panel (b)) and the PSD in the system at $\rho=0.65$. One can see that the behavior of the dispersion curves at this density is qualitatively equivalent to the case of $\rho=0.5$ : there is an anomalous region of temperatures where the excitation frequency decreases with temperature. A well pronounced minimum is observed at panel $(\mathrm{b})$ at $T \approx 0.5$. At the same time the PSD has complex dependence on temperature at temperatures from $T=1.0$ up to $T=1.5$. The PSD becomes smaller or larger with temperature. However, such irregular behavior of PSD can be also a result of inaccuracies of calculations.

In the case of $\rho=1.15$ the behavior of the dispersion curves is normal, i.e. frequencies increase with temperature. The corresponding plots are given in the supplementary materials.

Importantly, the anomalous behavior of the system under consideration is related to the presence of two length scales in the potential 1. A competition between these length scales takes place at intermediate densities 23 -

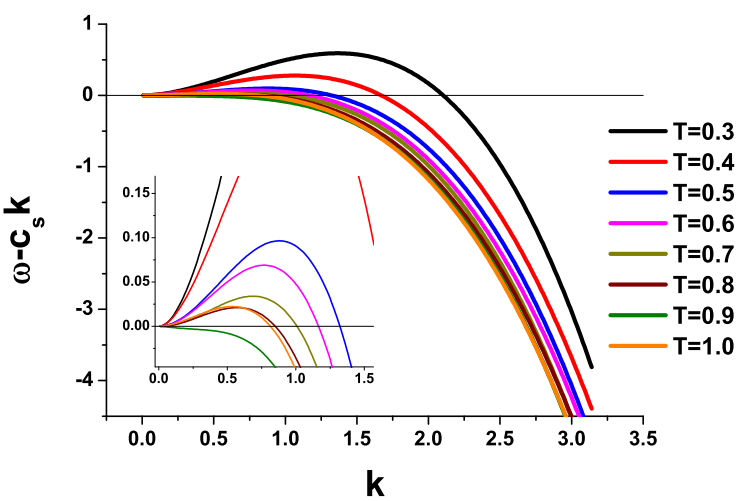

FIG. 3: PSD at $\rho=0.5$. The inset enlarges the region of small k. The horizontal line marks the zero level.

28]. The density $\rho=1.15$ is rather high and the behavior of the system is dominated by the core of the potential $\sigma$. Because of this no anomalies is expected at this density which is confirmed by the results of simulations.

The system with potential 1 demonstrates a number of water-like features, like, density anomaly, diffusion anomaly, structural anomaly, maxima of response functions, etc. Basing on this one can guess that presence of the dispersion curve anomaly in the RSS system can be also similar to the behavior of water and that water also demonstrates the dispersion curve anomaly. In order to check it we performed simulations of the SPC/E model of water. Since the simulation of water is more time-consuming we collected much less statistics and the spectra are much noisier comparing to the ones of RSS system. We employ the same MCT fitting of the spectra (eq. (4) in order to remove the noise. Several examples of dispersion curves are shown in Fig. 5. One can see that the MD results are rather noisy, but the fitting works quite well. Fig. [6] shows the dispersion curves of water at a set of temperatures along $\rho=1 \mathrm{~g} / \mathrm{cm}^{3}$ isochor. Only the results of fitting are shown. Comparing to the case of RSS model the dispersion curves look more complex. At low temperatures one can observe a bend of the curves downward at low $\mathrm{k}$ vectors which disappears at high temperatures and the curves intersect at intermediate wave vectors. Because of this we compare the frequencies at the highest wave vectors, which are close to the Brillouin zone boundary. In the case of liquids the boundary of Brillouin zone is a half of the wave vector corresponding to the first maximum of the structure factor. One can see that at the temperatures below $600 \mathrm{~K}$ the excitation frequencies decrease with temperature. Therefore, the same type of anomaly is observed in water too. However, this conclusion requires more elaborate calculations, since the dispersion curves of water are more complex then the ones of the RSS model. 


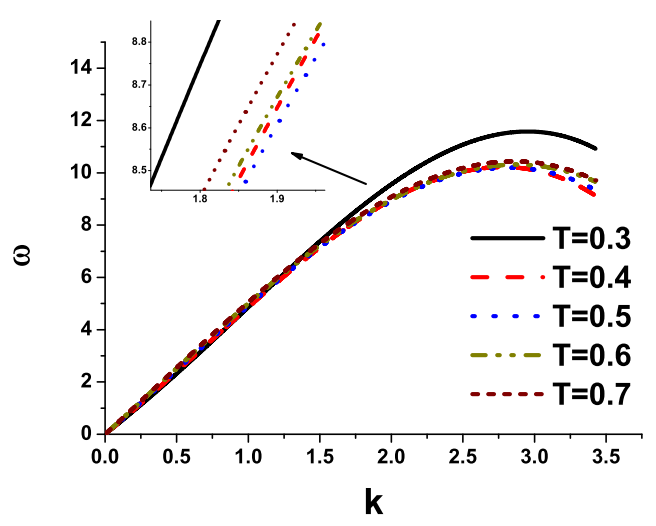

(a)

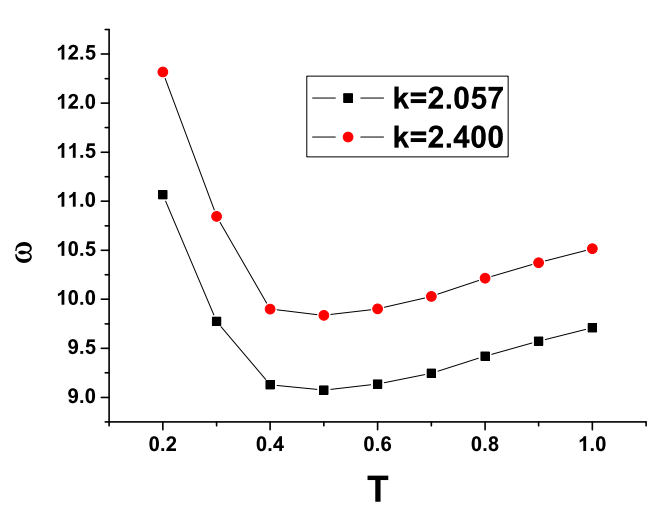

(b)

(c)

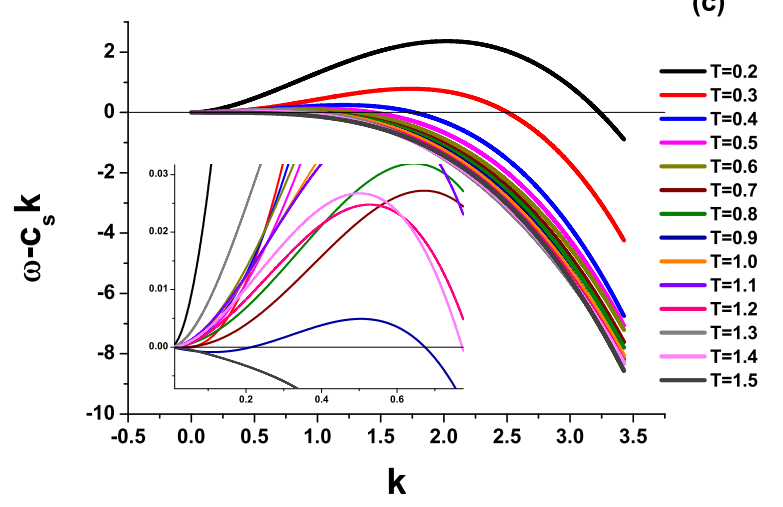

FIG. 4: (a) Dispersion curves at density $\rho=0.65$. The inset enlarges the region $k \approx 1.85$. (b) Temperature dependence of excitation frequency at fixed wave-vector. (c) PSD in the system.

As it was mentioned in Introduction, water demonstrates many different anomalies. The results of the present paper discover one more anomaly of water: anomalous dependence of the excitation frequencies on temperature.

In conclusion, we consider the spectra of longitudinal excitations in a core-softened system (RSS model) and we find that at some densities the dispersion curves demonstrate anomalous temperature dependence of the excitation frequencies. The same kind of anomaly is also

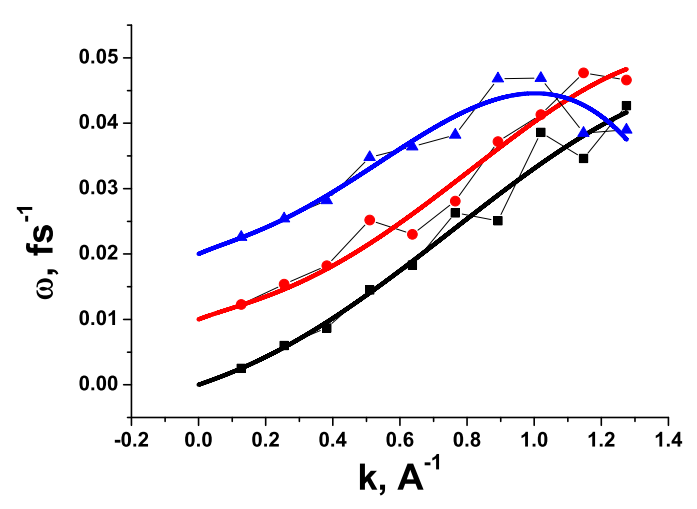

FIG. 5: Examples of dispersion curves of water and their approximation by MCT formula (eq. (4). From bottom to top: $T=400 \mathrm{~K}, 500 \mathrm{~K}$ and $600 \mathrm{~K}$. The curves are shifted by 0.01 with respect to the previous one in order to make the plot clearer. The symbols give the MD results and the curves the results of fitting.

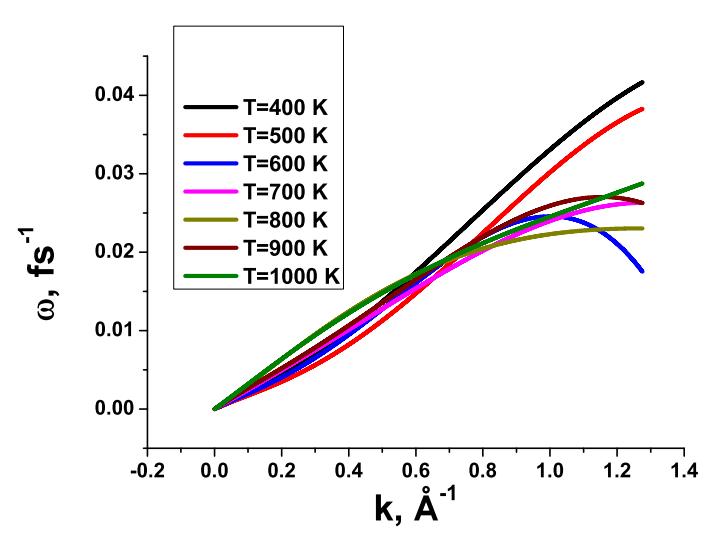

FIG. 6: Dispersion curves of water at $\rho=1 \mathrm{~g} / \mathrm{cm}^{3}$ and different temperatures.

observed in SPC/E model of water which allows to say thay a novel anomaly of water is discovered. Further investigations are required in order to find out the reasons of this anomaly.

This work was carried out using computing resources of the federal collective usage center "Complex for simulation and data processing for mega-science facilities" at NRC "Kurchatov Institute", http://ckp.nrcki.ru, and supercomputers at Joint Supercomputer Center of the Russian Academy of Sciences (JSCC RAS). The work was supported by the Russian Foundation of Basic Research (Grants No 18-02-00981).

[1] N. W. Ashcroft, and N. D. Mermin, Solid State Physics, Saunders Colledge Publishing (1976). 
[2] C. Kittel, Introduction to Solid State Physics, John Wiley (1953).

[3] J. P. Boon, S. Yip, Molecular Hydrodynamics, McGrawHill International Book Company, New York (1980)

[4] T. Scopigno, G. Ruocco, and F. Sette, Rev. Mod. Phys. 77,881 (2005).

[5] D. Bolmatov, V. V. Brazhkin, and K. Trachenko, Sci. Rep. 2012; 2, 421-427 (2012).

[6] Levesque D, Verlet L, and Kürkijarvi J, Phys. Rev. A 7, 1690 (1973).

[7] Hosokawa S, Inui M, Kajihara Y, Tsutsui S and Baron A Q R, J. Phys.: Cond. Matt. 27, 194104 (2015).

[8] Hosokawa S et. al., Eur. Phys. J. Special Topics 196, 8593 (2011)

[9] Hosokawa S, Inui M, Matsuda K, Ishikawa D, Baron A Q R, Journal of Physics: Conference Series 98 (2008) 022004

[10] Hosokawa S, Pilgrim W-C, Demmel F, Journal of NonCrystalline Solids 353 (2007) 31223128

[11] Hosokawa S, Inui M, Matsuda K, Ishikawa D and Baron A Q R, Phys. Rev. B 77, 174203 (2008)

[12] Hosokawa S et. al., J. Phys.: Condens. Matter 25 (2013) 112101

[13] Brazhkin V V et al, Phys. Rev. E 85, 031203 (2012).

[14] Brazhkin V V et al, Phys. Rev. Lett. 111, 145901 (2013).

[15] Brazhkin V V et al, Phys. Usp. 55, 1061 (2012).

[16] http://www1.lsbu.ac.uk/water/water nomalies.html

[17] C. Vega, J. L. F. Abascal, M. M. Conde and J. L. Aragones, 141, 251-276 (2009).

[18] C. Vega and J. L. F. Abascal, Phys. Chem. Chem. Phys. 13, 19663-19688 (2011).

[19] O. Mishima and H. Eugene Stanley, Nature 396, 329 (1998).

[20] C.N.Likos, H.M.Harreis, Condensed Matter Physics 5, 173-200 (2002).

[21] N. V. Gribova, Yu. D. Fomin, D. Frenkel and V. N. Ryzhov, Phys. Rev. E 79, 051202 (2009).

[22] J. R. Errington and P. G. Debenedetti, Nature 409, 318 (2001).

[23] M. Scott Shell, P. G. Debenedetti, and A. Z. Panagiotopoulos, Phys. Rev. E 66, 011202 (2002).

[24] Yu. D. Fomin, E. N. Tsiok, V. N. Ryzhov, Phys. Rev. E 87, $042122(2013)$.

[25] Yu.D. Fomin, E.N. Tsiok, and V.N. Ryzhov, Eur. Phys. J. Special Topics 216, 165173 (2013).

[26] D. Dhabal, Ch. Chakravarty, V. Molinero, and H. K. Kashyap, J. Chem. Phys. 145, 214502 (2016).

[27] J. L. F. Abascal and C. Vega, J. Chem. Phys. 123, 234505 (2005).

[28] Yu. D. Fomin, N. V. Gribova, V. N. Ryzhov, S. M. Stishov, and Daan Frenkel, J. Chem. Phys. 129, 064512 (2008).

[29] Yu. D. Fomin, E. N. Tsiok, and V. N. Ryzhov, J. Chem. Phys. 135, 234502 (2011).

[30] Yu. D. Fomin and V. N. Ryzhov, Physics Letters A 375 (2011) 21812184.

[31] Yu. D. Fomin, E. N. Tsiok, and V. N. Ryzhov, Eur. Phys. J. Special Topics 216, 165173 (2013).

[32] Yu. D. Fomin, E. N. Tsiok, and V. N. Ryzhov, J. Chem. Phys. 135, 124512 (2011).

[33] Daan Frenkel and Berend Smit, Understanding molecular simulation (From Algorithms to Applications), 2nd Edition (Academic Press, 2002).

[34] Hansen J P and McDonald I R, Theory of Simple Liquids
(Elsevier, 2013).

[35] Rapaport D C "The Art of Molecular Dynamics Simulation" Cambridge University Press (1995).

[36] M. H. Ernst, and J. R. Dorfman, Journal of Statistical Physics, 12, 311 (1975).

[37] Eu. A. Gaiduk, Yu. D. Fomin, V.N. Ryzhov, E.N. Tsiok, V.V. Brazhkin, Fluid Phase Equilibria, 417 (2016) 237241.

[38] http://lammps.sandia.gov/

[39] J. Hubbard and J. L. Beeby, J. of Physics C: Solid State Physics, 2, 556 (1969). 


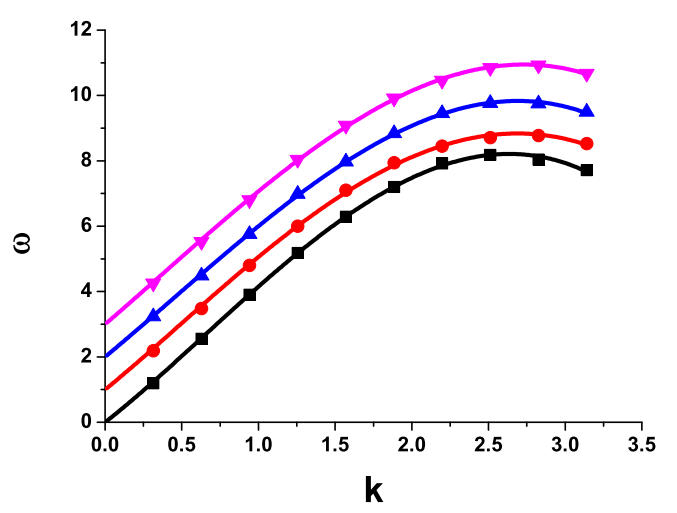

FIG. 7: Dispersion curves of the system at $\rho=0.5$ and (from bottom to top) $T=0.3,0.4,0.5$ and 0.6. Each upper curve is shifted by unity from the previous one to make the figure clearer. The symbols denote the data from simulation while the lines are the fitting by eq. 4 .

\section{ANOMALOUS BEHAVIOR OF DISPERSION CURVES IN WATER-LIKE SYSTEMS AND WATER: SUPPLEMENTARY MATERIALS.}

\section{FITTING OF THE DISPERSION CURVES}

As it is discussed in the main text, the dispersion curves are fitted by the Mode-Coupling Theory (MCT) equation:

$$
\omega(k)=c_{s} k+b_{1} k^{5 / 2}+b_{2} k^{11 / 4}+b_{3} k^{23 / 8},
$$

where $c_{s}$ is adiabatic speed of sound and $b_{1}, b_{2}$ and $b_{3}$ are fitting coefficients [1].

Here we show several dispersion curves obtained in the simulation and perform their fitting by eq. 4. The results are shown in Fig. 7 One can see that eq. 4 very accurately describes the dispersion curves obtained in the simulations. At the same time it efficiently removes the noise. Because of this we use only the fitted curves in the main text.

\section{HUBBARD-BEEBY MODEL APPROXIMATION}

The simplest approach to the excitation spectra in liquids is Hubbard-Beeby (HB) model [2]. Although this model does not give good quantitative agreement with simulation, usually it gives correct qualitative behavior. In Fig. 8 we give several dispersion curves obtained in frames of HB model. We also give the dispersion curve from this work for $T=0.3$ for comparison with the $\mathrm{HB}$ curve. One can see that the HB model overestimates the frequencies. But the most important is that the HB

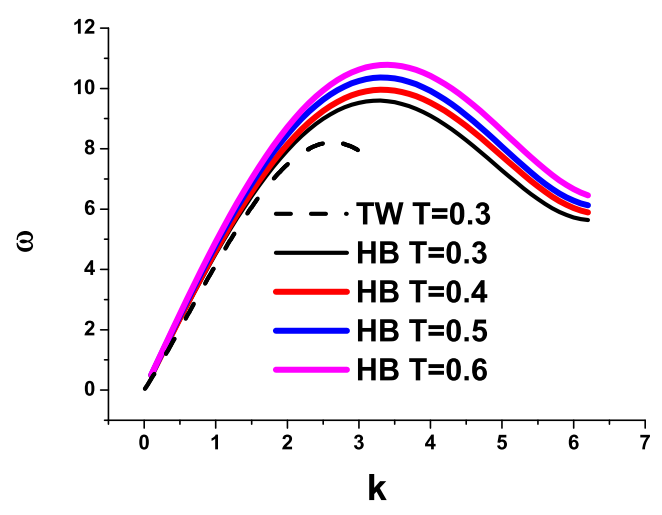

FIG. 8: Dispersion curves at $\rho=0.5$ and several temperatures obtained in Hubburd-Beeby approach. For $T=0.3$ we compare the results with the results from simulations. From bottom to top: $T=0.3$ this work (TW), $T=0.3 \mathrm{HB}, T=0.4$ $\mathrm{HB}, T=0.5 \mathrm{HB}, T=0.6 \mathrm{HB}$.

model does not give the decrease of frequencies with temperature for this system. Therefore HB model is not applicable to the liquids with anomalous temperature dependence of the dispersion curves. It is especially interesting because HB model is widely used to describe the excitation spectra in liquid metals (see, for instance, Refs. $3-6]$ and references therein). As it was shown in Refs. 7, 8] the effective interaction potentials in liquid metals are core-softened ones as the potential studied in the present work. Because of this one can expect that HB model gives inappropriate description of the dispersion curves of liquid metals. However, nowadays there are more elaborate theories which give much better description of the dispersion curves of liquids, such as Generalized Hydrodynamics introduced in Refs. [9, 10] (to name a few successful implementations see, for instance, $[11[13])$ and especially the theory which allows to calculate the dynamic structure factor with high precision introduced in Refs. [14 16] (see, Refs. [14 19] for some examples of successful implementaltion).

\section{DENSITY ANOMALY AND THE SPEED OF SOUND}

The SRS system at $\rho=0.5$ the system demonstrates the density anomaly [23 28] which leads to appearance of a minimum on the pressure dependence on temperature at this isochore. However, the adiabatic speed of sound $c_{s}$ along the same isochore is a monotonous function of temperature. The corresponding plots are given in Fig. Fig. 9 (a) and (b). 

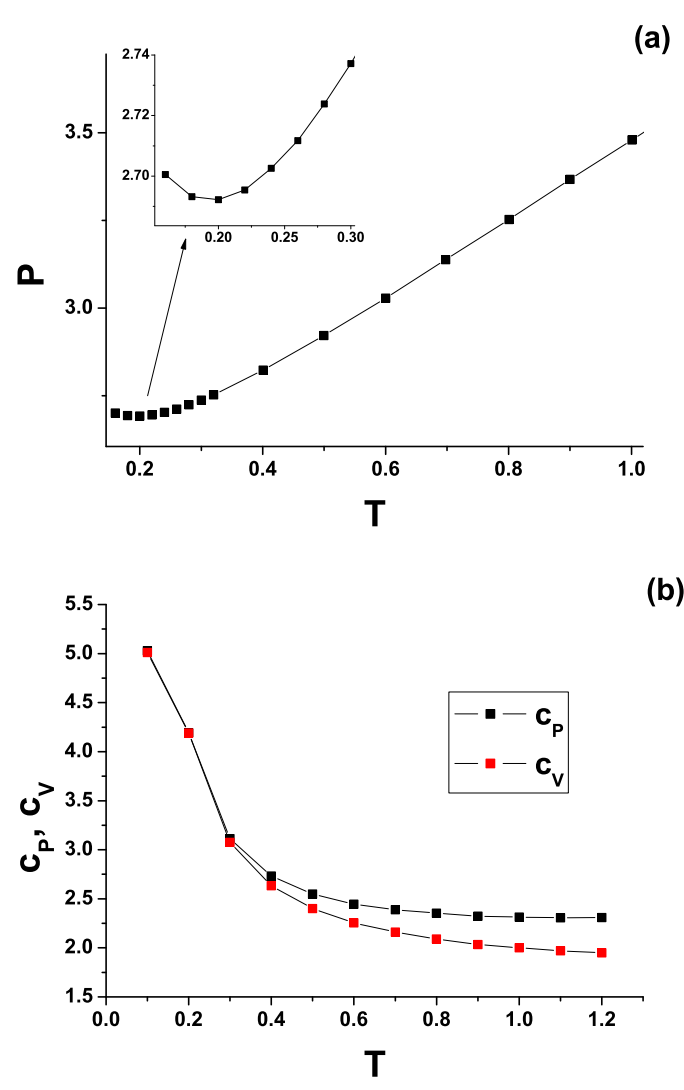

FIG. 9: (a) Equation of state along the isochore $\rho=0.5$. The inset enlarges the region of the density anomaly. (b) Adiabatic speed of sound at $\rho=0.5$. The symbols are the results of simulations and the line is the polynomial fit.

\section{SRS AT HIGH DENSITY}

As a final check of our results we calculate the dispersion curves of the SRS at high density, where no anomalies is expected. Fig. 10 shows the dispersion curves at $\rho=1.15$. One can see that the dispersion curves behave normally, i.e. the frequency increases with temperature. Therefore, the anomalous dependence of the excitation spectra takes place in the region of intermediate densities where a competition between different length scales takes place, but it disappears at the high density where the small length scale is prevailing.

[1] M. H. Ernst, and J. R. Dorfman, Journal of Statistical Physics, 12, 311 (1975).

[2] J. Hubbard and J. L. Beeby, J. of Physics C: Solid State Physics, 2, 556 (1969).

[3] A. M. Vora, Journal of Non-Oxide Glasses, 2, 91-96 (2010).

[4] N. H. March, K. N. Pathak, and J. A. Ascough Phys. Chem. Liq. 21, pp. 203-206 (1990).

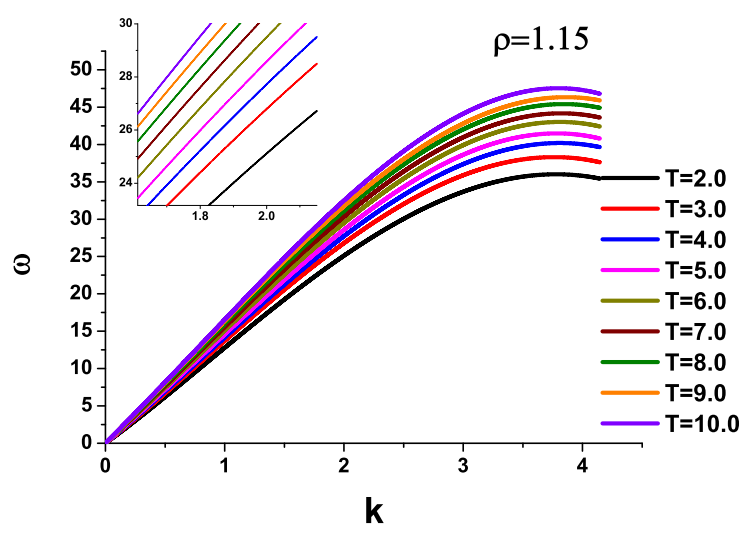

FIG. 10: Dispersion curves at density $\rho=1.15$. The inset enlarges the region about $k \approx 1.9$. One can see that the frequencies increase with temperature.

[5] K. N. Pathak and R. Bansal, J. Phys. C: Solid State Phys.6, 1989 (1973).

[6] V.N.Patel, P.B.Thakor, B.Y.Thakore, P.N.Gajjar, A.R.Jan, Condensed Matter Physics 9,pp. 74174 (2006).

[7] H. S. Kang, Phys. Rev. B. 60, 6362 (1999).

[8] J.-F. Wax, R. Albaki, and J.-L. Bretonnet, Phys. Rev. B 14, 14818 (2000).

[9] B. Kamgar-Parsi, E. G. D. Cohen, and I. M. de Schepper Phys. Rev. A 35, 4781 (1987).

[10] I. M. de Schepper, E. G. D. Cohen, C. Bruin, J. C. van Rijs, W. Montfrooij, and L. A. de Graaf Phys. Rev. A 38, 271 (1988).

[11] T. Bryk, I. Mryglod, T. Scopigno, G. Ruocco, F. Gorelli, and M. Santoro J. Chem. Phys. 133, 024502 (2010).

[12] T Bryk and A. B. Belonoshko, Phys. Rev. B 86, 024202 (2012).

[13] T.Bryk, I.Mryglod, Condensed Matter Physics, 6, 395 (2003).

[14] A.V. Mokshin, R.M. Yulmetyev, P. Hanggi, V.Yu. Shurygin, Phys. Rev. E 64 057101(1) - 057101 (2001).

[15] A.V. Mokshin, R.M. Yulmetyev, T. Scopigno, P. Hanggi, J. Phys.: Condens. Matter 15, 2235 (2003).

[16] A.V. Mokshin, Theor. Math. Phys. 183, 449 (2015).

[17] Yulmetyev R.M, Mokshin A.V, Hanggi P, Shurygin V.Y. JETP Letters. - 2002. - Vol.76, Is.3. - P.147-150.

[18] R.M. Yulmetyev, A.V. Mokshin, P. Hanggi, V.Yu. Shurygin, JETP Letters. - 2002. - v.76. - 147-151.

[19] A.V. Mokshin, R.M. Yulmetyev, P. Hanggi, J. Chem. Phys. - 2004. - Vol.121, No.15. - P.7341-7346.

[20] A. V. MokshinR. M. YulmetyevR. M. Khusnutdinov, P. Hanggi, ournal of Experimental and Theoretical Physics. - 2006. - v.103(6). - p.841-849.

[21] A.V. Mokshin, R.M. Yulmetyev, R.M. Khusnutdinov, P. Hanggi // Journal of Physics: Condensed Matter - 2007. - v.19. - p.046209(1)-046209(16).

[22] A. V. Mokshin, B. N. Galimzyanov // J. Phys.: Condens. Matter. - 2018. - vol. 30. - p. 085102.

[23] Yu. D. Fomin, N. V. Gribova, V. N. Ryzhov, S. M. Stishov, and Daan Frenkel, J. Chem. Phys. 129, 064512 (2008).

[24] N. V. Gribova, Yu. D. Fomin, N. V. Gribova, V. N. Ryzhov,and D. Frenkel, Phys. Rev. E 79, 051202 (2009). 
[25] Yu. D. Fomin, E. N. Tsiok, and V. N. Ryzhov, J. Chem. Phys. 135, 234502 (2011).

[26] Yu. D. Fomin and V. N. Ryzhov, Physics Letters A 375 (2011) 21812184.

[27] Yu. D. Fomin, E. N. Tsiok, and V. N. Ryzhov, Eur. Phys.
J. Special Topics 216, 165173 (2013).

[28] Yu. D. Fomin, E. N. Tsiok, and V. N. Ryzhov, J. Chem. Phys. 135, 124512 (2011). 